\title{
The use of Task Shifting in the Implementation of Prevention of Mother to Child Transmission of HIV (PMTCT) Services in Cameroon; the Option B+ Experience
}

\author{
Article by Nguosi Wam Joel \\ CBC Health Services, MPH Student, Texila American University, Cameroon \\ E-mail:wamjoel@yahoo.com
}

\begin{abstract}
Interventions to Prevent Mother to Child Transmission in the world have evolved over the years. Cameroon like every other country has been conforming to new recommendations from WHO based on evidence for better quality of service. Option B+ which entails provision of lifelong Antiretroviral Therapy (ART) to each pregnant and breastfeeding woman was recommended as the standard for PMTCT in 2012(1). Cameroon adopted these guidelines and set out for implementation in October 2013. The purpose of this study is to evaluate the effectiveness of task shifting from nurses to doctors in the rapid scale up of option $B+$ in Cameroon. Results show that within a period of fifteen months, a total of 520 nurses from 266 PMTCT sites in the South West region were trained on the provision of ART at MCH (maternal and child health) units. They were able to adequately provide ART to 1,754 women by December 2015 (Mvogo, 2016). These results set the pace for the scale up of Option B+ to other sites and regions across the country without putting pressure on the limited number of doctors in the country.
\end{abstract}

Keywords: Task shifting, PMTCT, Option B+, ART, MCH, HIV.

\section{Introduction}

The health system of Cameroon has over the years been facing the problem of inadequate staff in all the health professions; doctors, nurses, health technicians etc. The nurse to patient ratio is very big (16 per 10,000 patients) while the doctor to patient ratio is even worse ( 1 to 10,000 in urban areas and 1 to 40,000 in rural areas) (WHO, 2016). The distribution of staff amongst the existing health institutions is sometimes done with a lot of bias, influenced by political affiliations and some degree of corruption. There are some health institutions in the towns and cities that are over-crowded with staff while others in the certain rural areas barely have a few staff who are not adequately trained to take care of the health needs of the populations they serve. These health units are mostly run by just one health personnel, usually a nurse, who provides minimum services and refers to the next level when necessary.

Before now, the decision to place an HIV positive patient on Antiretroviral Therapy (ART) could only be made by a physician or a Therapeutic committee after deliberations. Cameroon has population of 23,739,000 persons (CDC, April 2016), 5\% of which are pregnant women. The prevalence HIV in the general population is $4.3 \%$ which is even higher for pregnant women $9.2 \%$. The country had achieved a coverage rate of ART of $58 \%$ which is comparatively higher than that of the neighboring countries in the sub-region.

Option $\mathrm{B}^{+}$is a PMTCT strategy which requires that every pregnant woman or breastfeeding mother who test positive for HIV should be placed on lifelong ART. This is different from the other PMTCT regiments that have been implemented in the country before now which had different criteria for initiation, none of which demanded immediate initiation of lifelong ART. Cameroon adopted this WHO recommendation in 2013 as the standard for PMTCT with plans of gradually rolling out the implementation nationwide by 2017. The existing set up had just about 166 HIV-care and treatment centers in the whole country which were not well distributed across the geographical landscape of the country. Providing ART to all eligible option $\mathrm{B}^{+}$clients within the country under such a set up would practically be 
Texila International Journal of Public Health

Volume 4, Issue 4, Dec 2016

impossible or would take a very long time. The country had to decide to follow WHOs recommendation of task shifting for HIV management in resource limited settings. Task shifting is the process of delegating tasks from more specialized to less specialized health workers and has been proposed as one of several possible solutions to the dire human resource shortages facing the African health sector (WHO, 2008). There was therefore the need to empower nurses to be able to take care of this problem.

\section{Methods}

Before the start of Option B+, the approved regiment for PMTCT in Cameroon was Option A where positive pregnant women had to do a CD4 test and/or WHO clinical classification to determine which course of treatment to follow. Those who were eligible for ART (CD4 below 350 cells or WHO stage $3 \& 4$ ) were referred to the approved HIV care and treatment centers to be further evaluated and placed on ART, while those who were not eligible for ART received a single molecule ARV for the duration of the pregnancy and breastfeeding period. This was mainly to prevent mother to child transmission of HIV but had not benefits to the mother in terms of improving her health or reducing the risk of transmission of infection to her partner.

The implementation of Option $\mathrm{B}+$ was a new phenomenon that could not just be introduced in the current set up, considering the fact that ART was provided only at approved HIV care and treatment centers. The South west region alone has over 250 health facilities that provide maternal and child health (MCH) services spread over 18 health districts. Out of this number, just 15 were HIV care and treatment centers and covered only 11 of the 18 health districts.

The South West region was fortunate to be one amongst the two regions selected for Pilot phase of the option B+ implementation in Cameroon. 10 health units were selected from 1 health district (Kumba) and 12 others in the other region (North west). Before selection, and initial assessment was done using a standard tool to determine their capacity to provide ART at the MCH unit. Some of the key aspects assessed include: the availability of staff, space, medication management, laboratory services etc. Three out of the 10 sites were care and treatment centers.

\section{Trainings and mentorship}

At least two nurses and nurse/midwives from the $\mathrm{MCH}$ units who previously were providing PMTCT services (Option A) were selected from each of these health facilities for training. The underwent a five days intensive training on the following areas: Generalities about HIV/AIDS, Counseling, Classes of ARVs, indications and side effects, client monitoring, opportunistic infections and data management. The trainings were led by doctors and nurses from HIV care and treatment programs. Doctors from some of these units were selected and trained on clinical supervision so that they will serve as site supervisors to the selected sites. After the nurses were trained, they we sent to spend some days at HIV care and treatment centers so that they can be familiar with the procedures and services carried out there. This was followed by implementation at the site level. Thereafter, every pregnant or breastfeeding woman who was tested positive for HIV was counseled, assessed and placed on ART to be taken for life at the MCH units in all the selected sites. Also, other positive clients who were previously on Option A and still current in care were switched to Option B+. The decision to place each client on treatment was taken by the nurses themselves, ARVs prescribed and the clients continuously monitored for any adverse effects. They received regular clinical supervision from the assigned doctors and mentorship from the regional program coordinators.

\section{Continuous quality assurance}

All the clients enrolled during this pilot phase did at least a CD4 test upon initiation as well as other work up tests like Full Blood Counts (FBC) and liver function tests. This was to set a 
baseline that would be used for assessment of the quality of treatment they are receiving and their response. Follow up CD4 tests were also done at six months' intervals upon initiation. Clients were also followed up for any side effects and adverse drug reactions. A few who developed adverse effects were transferred out to care and treatment centers for better management. Peer educators were also recruited to provide on-going psychosocial support, ensure adherence to treatment and retention in care.

\section{Results}

The pilot phase had a target to enroll 600 new clients into option $\mathrm{B}+$ in all the 22 pilot sites. This target was achieved over a period of ten months (October 2013 to July 2014). All the clients enrolled were followed up closely by the nurses with good documentation of all the interventions and close monitoring drug side effects, adverse drug reactions, adherence to medication, biological and immunological monitoring (CD4) and infant testing. The preliminary results proved to be successful and thus set the pace for scale up to others health facilities and districts in the region. The first scale up phase targeted all District hospitals and other high volume sites. In the following months, Option B+ was scaled to all the health units providing $\mathrm{MCH}$ services in the South west and North west regions, while the other regions in the country are following suit. By December 2015, a total of 520 nurses had been trained to provide ART for PMTCT from over 266 health units in the South West region of Cameroon, with over 1,754 positive women receiving ART for PMTCT that year in the region.

\section{Discussions}

The clients who were placed on treatment showed great improvement on their CD4 counts at 6 months and 12months and also recorded negative babies tested within the period of follow up. The purpose of this paper was not to show the effectiveness of these treatment regiments but rather to show that task shifting is a faster way of scaling up ART especially for PMTCT. These results are comparable to the those documented by Morris B (2009) et al, in Lusaka Zambia. Even though an in depth study has not been carried to do a comparative analysis of the client response to the treatment based on viral suppression in clients enrolled and followed up by doctors within this period, preliminary results show little difference in outcome. Further research could be carried to find out a number of issues:

- Compare the benefits of ART on the PMTCT program in this region to the previous PMTCT regiment (option A).

- Compare viral suppression rates of CD4 improvement between clients enrolled at the $\mathrm{B}+$ sites by nurses to those that were done by doctors at the care and treatment centers within the same period

\section{Conclusions}

The success of the pilot phase of option $\mathrm{B}^{+}$in the selected sites in the North West and South West regions of Cameroon which made way for the scale up to the other sites in these regions and other parts of the country shows that task shifting is an effective strategy for expansion of ART in Cameroon. Considering the fact that mother to child transmission of HIV is the leading cause of new infections in children, quick and efficient ways of reaching every positive pregnant woman with interventions that can reduce or prevent this vertical transmission are highly recommended. Task shifting thus could just be the solution needed to scale up ART for all members of the population at the different health units closer to them. In order for the Test and Treat recommendation for HIV care in the general population to be successfully implemented, task shifting has to be introduced at all levels beginning from the health units to the community.

\section{Acknowledgements}

Special thanks go to all the nurses and doctors, actors and partners who were fully involved in the scale up program. 
Texila International Journal of Public Health

Volume 4, Issue 4, Dec 2016

\section{References}

[1] CDC. (April 2016). CDC in Cameroon. Atlanta: Center for Global Health.

[2] Mary B Morris, Bushimbwa Tambatamba Chapula, Benjamin H Chi, Albert Mwango, Harmony F Chi, Joyce Mwanza, Handson Manda, Carolyn Bolton, Debra S Pankratz, Jeffrey SA Stringer and Stewart E Reid. (2009). Use of task-shifting to rapidly scale-up HIV treatment services: experiences from Lusaka Zambia. BMC Health Services Research.

[3] Mvogo. (2016). Rapport Annuel 2015. Yaounde.

[4] WHO. (2008). Train, treat, retain. Task Shifting: rational redistribution of tasks among health workforce teams; global recommendations and guidelines. Geneva, Switzerland: WHO Document Production Services.

[5] WHO. (2016, October 11). Country Cooperation Strategy. Retrieved from World Health Organization: http://www.who.int/countries/en 